\title{
DOMAIN DECOMPOSITION SPECTRAL APPROXIMATIONS FOR AN EIGENVALUE PROBLEM WITH A PIECEWISE CONSTANT COEFFICIENT**
}

\author{
M. S. MIN ${ }^{\dagger}$ AND D. GOTTLIEB ${ }^{\dagger}$
}

\begin{abstract}
Consider a model eigenvalue problem with a piecewise constant coefficient. We split the domain at the discontinuity of the coefficient function and define the multidomain variational formulation for the eigenproblem. The discrete multidomain variational formulations are defined for Legendre-Galerkin and Legendre-collocation methods. The spectral rate of convergence of the approximate eigensolutions is proven for the Legendre-Galerkin method. The minmax principle is used for the convergence analysis.

The Legendre-collocation, Chebyshev-collocation, Legendre-collocation penalty, and Chebyshevcollocation penalty methods are also defined by using the multidomain approach, and their numerical results applied to the eigenproblem are demonstrated. The spectral convergence for the eigenvalues and eigenfunctions is confirmed for all the multidomain spectral techniques presented here.
\end{abstract}

Key words. discontinuous problems, multidomain variational formulation, minmax principle, domain decomposition, Legendre-Galerkin method, Legendre-collocation method, Legendrecollocation penalty method, Chebyshev-collocation method, Chebyshev-collocation penalty method

AMS subject classifications. 41A10, 41A25

DOI. $10.1137 /$ S0036142903423836

1. Introduction. We consider Maxwell's equations governing the electromagnetic wave propagation in periodically structured dielectric arrays cast as an eigenvalue problem. The dielectric function corresponding to the periodic arrays is represented by a periodically piecewise constant function.

The electromagnetic wave propagation in a periodic dielectric medium was first studied by Rayleigh in 1887, identifying the fact that there exists a narrow frequency gap prohibiting light propagation through one-dimensional periodic twinning planes. In 100 years later, the concepts of omnidirectional forbidden frequency gaps in two and three dimensions were introduced, leading to many subsequent developments in the fabrication, theory, and application of electromagnetic wave propagation to optical fibers [14], [3], [15], [23]. Computation has become a primary tool for carrying out frequency gap calculation for various periodic dielectric structures.

Numerous numerical studies have focused on predicting the forbidden eigenfrequencies accurately by solving Maxwell's equations in the frequency domain [1], [6], [17], [16]. However, numerical analysis has been lacking, and high-order methods have not been applied to such problems yet.

In [18], Fourier-Galerkin and Fourier-collocation methods are applied to a single domain, and their theoretical and numerical convergence studies for the eigensolutions are demonstrated. As a result of the presence of the discontinuity in the coefficient function in a single domain, the solution is only in $H_{p}^{2}$, and the rates of convergence of the eigensolutions by Fourier methods are between second order and third order.

\footnotetext{
* Received by the editors February 26, 2003; accepted for publication (in revised form) July 29, 2004; published electronically DATE. This work was supported by AFOSR grant F49620-02-1-0113, NSF grant DMS-020745, and HPC/F33615-01-C-1866.

http://www.siam.org/journals/sinum/x-x/42383.html

${ }^{\dagger}$ Division of Applied Mathematics, Brown University, Providence, RI (msmin@cfm.brown.edu, dig@cfm.brown.edu).
} 
In this paper, we apply domain decomposition techniques for spectral methods. We obtain spectrally accurate eigensolutions by using multidomain Legendre and Chebyshev approximations. Implementations in two dimensions are extended in [19].

In the multidomain approach, we split the domain into subdomains in order for the discontinuous coefficient function to be smooth in each subdomain, so that the solutions are infinitely smooth in each subdomain. Then we reformulate the problem in multidomain variational form. The finite-dimensional space for each subdomain is defined by the Legendre polynomials of finite degrees, and boundary and interface conditions are imposed strongly for the Legendre-Galerkin, Legendre-collocation, and Chebyshev-collocation methods and weakly for the Legendre-collocation penalty and Chebyshev-collocation penalty methods.

We restrict the penalty parameter to a specific one in this paper and leave the study for the proper range of the parameters to future work. Convergence analysis for the eigenvalues and eigenfunctions is carried out for the Legendre-Galerkin method. For the collocation cases, two different methods are introduced by choosing two different test spaces for the same trial space.

The numerical results for Legendre-Galerkin, Legendre-collocation, Legendrecollocation penalty, Chebyshev-collocation, and Chebyshev-collocation penalty methods presented here show a spectral rate of convergence for the eigensolutions. In terms of accuracy, the results of the Legendre-Galerkin and the Legendre-collocation methods, which use the same space for the test and trial spaces, are comparable and more accurate than the results of the Legendre-collocation penalty and the Legendrecollocation methods, which use different spaces for the trial and test space. The penalty method is favorable because of the simplicity in implementation for the same magnitude of accuracy.

We organize this paper as follows. In section 2 we reformulate the eigenproblem into a multidomain variational formulation. We recall the minmax principle to characterize the $l$ th eigenvalue by minimizing the maximum of the Rayleigh quotient over $l$-dimensional subspaces. In section 3 we present the finite-dimensional space used for the approximate solution. The procedure to find the basis for the finite approximant space is shown. In section 4 we define the multidomain variational formulation for Legendre-Galerkin method. We provide a convergence analysis for the eigenvalues and eigenfunctions. The theory is confirmed by numerical results. In section 5 two different Legendre-collocation methods are defined, based on the test space chosen. The numerical results for eigensolutions by those methods show a spectral rate of convergence. In section 6 , we discuss the Legendre-collocation penalty method by defining a multidomain variational formulation with the penalty approach [7] for the boundary and interface constraints. Chebyshev-collocation approximations are also tested and their numerical results presented. Section 7 discusses the asymptotic behavior of the largest approximate eigenvalues. Section 8 gives a brief conclusion.

2. The multidomain variational formulation. The source-free Maxwell equations describing the transverse-magnetic mode in one-dimensional periodic media can be cast as the following generalized eigenvalue problem: find $\lambda$ and $u$ in $H_{p}^{2}(-\pi, \pi)$ (where $p$ stands for periodic), such that

$$
-u^{\prime \prime}=\lambda \epsilon(x) u,
$$

where $\epsilon(x)=1$ in $(-\pi, 0)$ and $\epsilon(x)=\omega^{2}$ in $[0, \pi), \omega \neq 1$. The function $u$ represents the electric field pattern, and the dielectric function $\epsilon(x)$ describes a unit cell from a multilayer structure with $2 \pi$-periodicity. This problem was considered in [18]. 
Recall the variational formulation of (2.1) from [18]: Find $\lambda$ and $u \in H_{p}^{1}(-\pi, \pi)$ such that

$$
a(u, v)=\lambda(u, v) \quad \text { for } v \in H_{p}^{1}(-\pi, \pi),
$$

where

$$
a(u, v)=\int_{-\pi}^{\pi} u^{\prime} v^{\prime} d x \quad \text { and } \quad(u, v)=\int_{-\pi}^{\pi} u v \epsilon d x .
$$

Since $a(u, v)$ is Hermitian, the eigenvalue can be characterized by the following two statements from [9], [18], [20], [21].

ThEOREm 2.1. Let $\lambda_{l}$ denote the eigenvalues of (2.1), and let $S_{l}$ be any $l$ dimensional subspace of $H_{p}^{1}(\Omega)$. Then, for $\lambda_{1} \leq \lambda_{2} \leq \cdots \leq \lambda_{l} \cdots$,

$$
\lambda_{l}=\min _{S_{l} \subset H_{p}^{1}(\Omega)} \max _{v \in S_{l}} \frac{a(v, v)}{(v, v)} .
$$

We also recall the following lemma from [9] and [18].

LEMMA 2.2. Let $\lambda_{i}$ be arranged in ascending order, and define

$$
E_{i, j}=\operatorname{span}\left\{u_{i}, \ldots, u_{j}\right\},
$$

where $u_{i}$ is the eigenfunction corresponding to the eigenvalue $\lambda_{i}$. Then

$$
\begin{aligned}
& \lambda_{l}=\max _{v \in E_{k, l}} \frac{a(v, v)}{(v, v)}, \quad k \leq l, \\
& \lambda_{l}=\min _{v \in E_{l, m}} \frac{a(v, v)}{(v, v)}, \quad l \leq m .
\end{aligned}
$$

It is natural here to consider splitting the domain. Denote the domain by $\Omega=$ $(-\pi, \pi)$ and divide it into two subdomains, say, $\Omega_{-}=(-\pi, 0)$ and $\Omega_{+}=(0, \pi)$, so that $\epsilon(x)$ is smooth in each subdomain. Denote the restrictions by $u_{-}=\left.u\right|_{\Omega_{-}}$and $u_{+}=\left.u\right|_{\Omega_{+}}$, which are distributional solutions to the given equation (2.1). Integrate by parts in $\Omega_{1}$ and $\Omega_{2}$, respectively, and define the following bilinear forms:

$$
\begin{array}{ll}
a(u, v)_{-}=\int_{-\pi}^{0} u_{-}^{\prime} v_{-}^{\prime} d x+\left.u_{-}^{\prime} v_{-}\right|_{0} ^{-\pi}, & (u, v)_{-}=\int_{-\pi}^{0} u_{-} v_{-} \epsilon_{-} d x, \\
a(u, v)_{+}=\int_{0}^{\pi} u_{+}^{\prime} v_{+}^{\prime} d x+\left.u_{+}^{\prime} v_{+}\right|_{\pi} ^{0}, & (u, v)_{+}=\int_{0}^{\pi} u_{+} v_{+} \epsilon_{+} d x .
\end{array}
$$

Remark 1. It is clear to see that, for $u, v \in H_{p}^{1}(\Omega)$,

$$
\begin{aligned}
a(u, v) & =a(u, v)_{-}+a(u, v)_{+}, \\
(u, v) & =(u, v)_{-}+(u, v)_{+} .
\end{aligned}
$$

3. Finite-dimensional subspace. In this section we present the finite-dimensional space used in our approximation. Denote by $\mathcal{P}_{N}=\operatorname{span}\left\{L_{k}(\xi), \xi \in[-1,1]\right\}$ the space of Legendre polynomials of degree at most $N$. We define the local variables $x_{-}$and $x_{+}$by

$$
x_{-}(\xi)=\frac{\pi}{2}(\xi-1) \text { and } \quad x_{+}(\xi)=\frac{\pi}{2}(\xi+1) .
$$


The approximation space $V_{2 N-2}$ is the $(2 N-2)$-dimensional space defined by

$$
V_{2 N-2}=\left\{\phi \in H_{p}^{2}(\Omega): \phi_{-}\left(x_{-}(\xi)\right) \in \mathcal{P}_{N} \text { and } \phi_{+}\left(x_{+}(\xi)\right) \in \mathcal{P}_{N}\right\} .
$$

To apply the Galerkin approximation, we need to find a basis of $V_{2 N-2}$. This is done as follows.

Let $\phi$ in $V_{2 N-2}$ be expressed by

$$
\begin{aligned}
& \phi_{-}=\left.\phi\right|_{x_{-}(\xi)}=\sum_{k=0}^{N} c_{k} L_{k}(\xi), \\
& \phi_{+}=\left.\phi\right|_{x_{+}(\xi)}=\sum_{k=0}^{N} d_{k} L_{k}(\xi) .
\end{aligned}
$$

Since $\phi$ and $\phi^{\prime}$ are continuous at $x=0$ and $2 \pi$-periodic, we apply the following conditions:

$$
\begin{aligned}
& \phi_{-}(0)=\phi_{+}(0), \phi_{-}(-\pi)=\phi_{+}(\pi), \\
& \phi_{-}^{\prime}(0)=\phi_{+}^{\prime}(0), \phi_{-}^{\prime}(-\pi)=\phi_{+}^{\prime}(\pi) .
\end{aligned}
$$

Letting $\alpha_{k}=L_{k}^{\prime}(1)=\frac{k(k+1)}{2}$, and applying the boundary and the interface conditions (3.5) and (3.6), we obtain the following relations for the coefficients:

$$
\begin{aligned}
c_{N} & =\sum_{k=1}^{\frac{N-2}{2}}\left[-\frac{\left(\alpha_{N}+\alpha_{2 k}\right)}{2 \alpha_{N}} c_{2 k}+\frac{\left(\alpha_{N}-\alpha_{2 k}\right)}{2 \alpha_{N}} d_{2 k}\right], \\
d_{N} & =\sum_{k=1}^{\frac{N-2}{2}}\left[\frac{\left(\alpha_{N}-\alpha_{2 k}\right)}{2 \alpha_{N}} c_{2 k}-\frac{\left(\alpha_{N}+\alpha_{2 k}\right)}{2 \alpha_{N}} d_{2 k}\right], \\
c_{N-1} & =\sum_{k=0}^{\frac{N-4}{2}}\left[-\frac{\left(\alpha_{N-1}+\alpha_{2 k+1}\right)}{2 \alpha_{N-1}} c_{2 k+1}-\frac{\left(\alpha_{N-1}-\alpha_{2 k+1}\right)}{2 \alpha_{N-1}} d_{2 k+1}\right], \\
d_{N-1}= & \sum_{k=0}^{\frac{N-4}{2}}\left[-\frac{\left(\alpha_{N-1}-\alpha_{2 k+1}\right)}{2 \alpha_{N-1}} c_{2 k+1}-\frac{\left(\alpha_{N-1}+\alpha_{2 k+1}\right)}{2 \alpha_{N-1}} d_{2 k+1}\right] .
\end{aligned}
$$

For simplicity, here we use the following notation:

$$
\begin{gathered}
\beta_{k}=\frac{\alpha_{N_{k}}+\alpha_{k}}{2 \alpha_{N_{k}}} \quad \text { and } \quad \gamma_{k}=(-1)^{k} \frac{\alpha_{N_{k}}-\alpha_{k}}{2 \alpha_{N_{k}}}, \\
N_{k}=\left\{\begin{array}{cl}
N & \text { for even } k, \\
N-1 & \text { for odd } k .
\end{array}\right.
\end{gathered}
$$

Then, substituting (3.7)-(3.10) into (3.3)-(3.4), we get

$$
\begin{aligned}
& \phi_{-}=\left.\phi\right|_{x_{-}(\xi)}=\sum_{k=0}^{N-2} c_{k}\left\{L_{k}(\xi)-\beta_{k} L_{N_{k}}(\xi)\right\}+\sum_{k=0}^{N-2} d_{k} \gamma_{k} L_{N_{k}}(\xi), \\
& \phi_{+}=\left.\phi\right|_{x_{+}(\xi)}=\sum_{k=0}^{N-2} c_{k} \gamma_{k} L_{N_{k}}(\xi)+\sum_{k=0}^{N-2} d_{k}\left\{L_{k}(\xi)-\beta_{k} L_{N_{k}}(\xi)\right\} .
\end{aligned}
$$


Thus, one can easily see the basis for $V_{2 N-2}$ given by

$$
\left\{\phi_{k}, \psi_{k}\right\} \quad \text { for } 0 \leq k \leq N-2
$$

where the basis functions are defined by

$$
\begin{aligned}
& \left(\phi_{k}\right)_{-}=L_{k}(\xi)-\beta_{k} L_{N_{k}}(\xi), \quad\left(\phi_{k}\right)_{+}=\gamma_{k} L_{N_{k}}(\xi), \\
& \left(\psi_{k}\right)_{-}=\gamma_{k} L_{N_{k}}(\xi), \quad\left(\psi_{k}\right)_{+}=L_{k}(\xi)-\beta_{k} L_{N_{k}}(\xi) .
\end{aligned}
$$

Now, we are ready to construct the multidomain Legendre-Galerkin scheme in the following section.

4. Legendre-Galerkin method. Find $\lambda^{N}, u^{N} \in V_{2 N-2}$ such that

$$
a\left(u^{N}, v^{N}\right)=\lambda^{N}\left(u^{N}, v^{N}\right) \quad \text { for all } v^{N} \in V_{2 N-2} \text {. }
$$

From the relations (2.10) and (2.11), the two inner products can be expressed by

$$
\begin{aligned}
& a\left(u^{N}, v^{N}\right)=\int_{-\pi}^{0}\left(u_{-}^{N}\right)^{\prime}\left(v_{-}^{N}\right)^{\prime} d x+\int_{0}^{\pi}\left(u_{+}^{N}\right)^{\prime}\left(v_{+}^{N}\right)^{\prime} d x=\int_{-\pi}^{\pi}\left(u^{N}\right)^{\prime}\left(v^{N}\right)^{\prime} d x \\
& \left(u^{N}, v^{N}\right)=\int_{-\pi}^{0}\left(u_{-}^{N}\right)\left(v_{-}^{N}\right)\left(\epsilon_{-}\right) d x+\int_{0}^{\pi}\left(u_{+}^{N}\right)\left(v_{+}^{N}\right)\left(\epsilon_{+}\right) d x=\int_{-\pi}^{\pi} u^{N} v^{N} \epsilon d x
\end{aligned}
$$

4.1. Numerical scheme and its results. The approximate eigenfunction $u^{N} \in$ $V_{2 N-2}$ can be expanded by the basis found in section 3 with an unknown set of $(2 N-2)$ coefficients:

$$
u^{N}=\sum_{k=0}^{N-2}\left[\left(\hat{u}_{\phi}^{N}\right)_{k} \phi_{k}+\left(\hat{u}_{\psi}^{N}\right)_{k} \psi_{k}\right]
$$

Take $v^{N}=\phi_{n}$ for $0 \leq n \leq N-2$, and substitute $u^{N}$ in the form (4.4) to the variational formulation (4.2)-(4.3). We obtain the Legendre-Galerkin scheme as follows:

$$
K \hat{\mathbf{u}}^{N}=\lambda^{N} M \hat{\mathbf{u}}^{N}
$$

The following are defined for the notation in scheme (4.5):

$$
K=\left[\begin{array}{cc}
K_{-}^{-} & K_{+}^{-} \\
K_{-}^{+} & K_{+}^{+}
\end{array}\right], \quad M=\left[\begin{array}{cc}
M_{-}^{-} & M_{+}^{-} \\
M_{-}^{+} & M_{+}^{+}
\end{array}\right], \text {and } \hat{\mathbf{u}}^{N}=\left[\begin{array}{c}
\hat{u}_{\phi}^{N} \\
\hat{u}_{\psi}^{N}
\end{array}\right] \text {, }
$$

where

$$
\begin{array}{ll}
\left(K_{-}^{-}\right)_{k, n}=\int_{-\pi}^{\pi}\left(\phi_{k}\right)^{\prime}\left(\phi_{n}\right)^{\prime} d x, & \left(K_{+}^{-}\right)_{k, n}=\int_{-\pi}^{\pi}\left(\phi_{k}\right)^{\prime}\left(\psi_{n}\right)^{\prime} d x, \\
\left(K_{-}^{+}\right)_{k, n}=\int_{-\pi}^{\pi}\left(\psi_{k}\right)^{\prime}\left(\phi_{n}\right)^{\prime} d x, & \left(K_{+}^{+}\right)_{k, n}=\int_{-\pi}^{\pi}\left(\psi_{k}\right)^{\prime}\left(\psi_{n}\right)^{\prime} d x, \\
\left(M_{-}^{-}\right)_{k, n}=\int_{-\pi}^{\pi} \phi_{k} \phi_{n} \epsilon(x) d x, & \left(M_{+}^{-}\right)_{k, n}=\int_{-\pi}^{\pi} \phi_{k} \psi_{n} \epsilon(x) d x, \\
\left(M_{-}^{+}\right)_{k, n}=\int_{-\pi}^{\pi} \psi_{k} \phi_{n} \epsilon(x) d x, & \left(M_{+}^{+}\right)_{k, n}=\int_{-\pi}^{\pi} \psi_{k} \psi_{n} \epsilon(x) d x,
\end{array}
$$


TABLE 1

Relative errors of eigenvalues for $\omega=2$ and the discrete $l_{2}$-errors of $u_{i}-u_{i}^{N}$ for the multidomain Legendre-Galerkin method.

\begin{tabular}{|c||c||c|c|}
\hline$\lambda_{i}$ & $N$ & $\left(\lambda_{i}^{N}-\lambda_{i}\right) / \lambda_{i}$ & $\left\|u_{i}-u_{i}^{N}\right\|$ \\
\hline & 4 & $2.94(-03)$ & $2.61(-03)$ \\
0.369875 & 8 & $7.04(-10)$ & $4.38(-07)$ \\
& 16 & $6.15(-15)$ & $1.03(-15)$ \\
& 32 & $7.38(-14)$ & $3.49(-15)$ \\
& 64 & $6.40(-13)$ & $2.55(-14)$ \\
\hline \multirow{5}{*}{0.536233} & 4 & $4.39(-04)$ & $8.77(-03)$ \\
& 8 & $1.63(-10)$ & $2.38(-06)$ \\
& 16 & $2.89(-14)$ & $2.45(-14)$ \\
& 32 & $3.43(-14)$ & $3.60(-14)$ \\
& 64 & $9.31(-14)$ & $1.42(-13)$ \\
\hline & 4 & $3.55(-02)$ & $1.71(-01)$ \\
& 8 & $1.75(-06)$ & $4.16(-04)$ \\
& 16 & $2.76(-16)$ & $5.43(-11)$ \\
& 32 & $5.94(-15)$ & $4.82(-14)$ \\
& 64 & $3.96(-14)$ & $2.85(-13)$ \\
\hline \multirow{5}{*}{1.937181} & 4 & $3.28(-01)$ & $1.05(-01)$ \\
& 8 & $1.45(-04)$ & $4.71(-04)$ \\
& 16 & $6.07(-15)$ & $2.31(-10)$ \\
& 32 & $2.64(-14)$ & $8.45(-15)$ \\
& 64 & $2.84(-13)$ & $8.09(-14)$ \\
\hline
\end{tabular}

and

$$
\hat{u}_{\phi}^{N}=\left[\left(\hat{u}_{\phi}^{N}\right)_{0},\left(\hat{u}_{\phi}^{N}\right)_{1}, \ldots,\left(\hat{u}_{\phi}^{N}\right)_{N-2}\right]^{T} \text { and } \hat{u}_{\psi}^{N}=\left[\left(\hat{u}_{\psi}^{N}\right)_{0},\left(\hat{u}_{\psi}^{N}\right)_{1}, \ldots,\left(\hat{u}_{\psi}^{N}\right)_{N-2}\right]^{T} .
$$

Now, we solve the generalized matrix eigenproblem (4.5) numerically and obtain the approximate $l(\leq 2 N-2)$ th eigenvalues, $\lambda_{l}^{N}$, and the set of orthogonal vectors $\hat{\mathbf{u}}^{N}$, which approximates the $l$ th eigenfunction $u_{l}$ as the coefficients in the expansion of the basis of $V_{2 N-2}$. In Table 1, the relative errors for $\lambda_{l}^{N}-\lambda_{l}$ and the discrete $l_{2}$-errors of $u_{l}-u_{l}^{N}$ as $N$ increases are provided for the first few eigenvalues in an ascending order and the associated eigenfunctions. The numerical results demonstrate that the errors decay exponentially as $N$ increases.

4.2. Error estimates for eigenvalues and eigenfunctions. We show the error estimates for the approximate eigenvalues and eigenfunctions for the multidomain Legendre-Galerkin method.

We first treat the approximate eigenvalues. Let $P_{N} u$ be defined by

$$
P_{N} u=\sum_{k=0}^{N-2}\left[\left(\hat{u}_{\phi}\right)_{k} \phi_{k}+\left(\hat{u}_{\psi}\right)_{k} \psi_{k}\right],
$$

where the expansion coefficients $\left(\hat{u}_{\phi}\right)_{k}$ and $\left(\hat{u}_{\psi}\right)_{k}$ will be defined later in this section.

From the minmax principle [9], [21], we can characterize the eigenvalue for the multidomain Legendre-Galerkin procedure by

$$
\lambda_{l}^{N}=\min _{S_{l} \subset V_{2 N-2}} \max _{v \in S_{l}} \frac{a(v, v)}{(v, v)} .
$$


Lemma 4.1. Let $\lambda_{l}^{N}$ be the approximation to $\lambda_{l}$ as obtained by the LegendreGalerkin procedure (4.5), and let $P_{N} u$ be defined as in (4.7). Then

$$
\lambda_{l} \leq \lambda_{l}^{N} \leq \lambda_{l} \max _{v \in E_{1, l}} \frac{a\left(P_{N} v, P_{N} v\right)}{a(v, v)} \max _{v \in E_{1, l}} \frac{(v, v)}{\left(P_{N} v, P_{N} v\right)} .
$$

Proof. Since $V_{2 N-2}$ is a subspace of $H_{p}^{1}(\Omega)$, it is true that $\lambda_{l} \leq \lambda_{l}^{N}$. Now, let $P E_{1, l}$ be spanned by $P_{N} u_{1}, \ldots, P_{N} u_{l}$. For simplicity, we denote by $P u=P_{N} u$. Clearly $P E_{1, l}$ is the $l$-dimensional subspace of $V_{2 N-2}$. Using the minmax principle, we have

$$
\begin{aligned}
\lambda_{l}^{N} & \leq \max _{v \in P E_{1, l}} \frac{a(v, v)}{(v, v)} \\
& =\max _{v \in E_{1, l}} \frac{a(P v, P v)}{(P v, P v)} \\
& =\max _{v \in E_{1, l}} \frac{a(v, v)}{(v, v)} \frac{a(P v, P v)}{a(v, v)} \frac{(v, v)}{(P v, P v)} .
\end{aligned}
$$

From Lemma 2.2, the proof follows.

Lemma 4.2. For $u_{i=1, \ldots, l} \in H_{p}^{1}(\Omega)$, where $\left(u_{i}\right)_{-} \in H^{m}\left(\Omega_{-}\right)$and $\left(u_{i}\right)_{+} \in$ $H^{m}\left(\Omega_{+}\right)$,

$$
\max _{v \in E_{1, l}} \frac{(v, v)}{(P v, P v)} \leq 1+C(l) N^{-m}
$$

where the constant $C(l)$ is independent of $N$.

Proof. We follow the procedure in [18]. For $v=\sum_{i=1}^{l} \mu_{i} u_{i}$ in $E_{1, l}$, we have

$$
\begin{aligned}
\frac{(v, v)-(P v, P v)}{(v, v)} & \leq \frac{2|(v, v-P v)|}{(v, v)} \\
& \leq \frac{2 \sum_{i, j=1}^{l}\left|\mu_{i}\right|\left|\mu_{j}\right|\left|\left(u_{i}-P u_{i}, u_{j}\right)\right|}{\sum_{i=1}^{l}\left|\mu_{i}\right|^{2}} \\
& =2 l \max _{i, j=1, \ldots, l}\left|\left(u_{i}-P u_{i}, u_{j}\right)\right| .
\end{aligned}
$$

For the last term above, we have

$$
\left|\left(u_{i}-P u_{i}, u_{j}\right)\right| \leq\left|\left(u_{i}-P u_{i}, u_{j}\right)_{-}\right|+\left|\left(u_{i}-P u_{i}, u_{j}\right)_{+}\right| .
$$

Now consider an eigenfunction $u=u_{i}$ and its projection $P u$ onto the space $V_{2 N-2}$. Since

$$
\begin{aligned}
P u_{-} & =\sum_{k=0}^{N-2}\left[\left(\hat{u}_{\phi}\right)_{k}\left(\phi_{k}\right)_{-}+\left(\hat{u}_{\psi}\right)_{k}\left(\psi_{k}\right)_{-}\right], \\
P u_{+} & =\sum_{k=0}^{N-2}\left[\left(\hat{u}_{\phi}\right)_{k}\left(\phi_{k}\right)_{+}+\left(\hat{u}_{\psi}\right)_{k}\left(\psi_{k}\right)_{+}\right],
\end{aligned}
$$

we can rewrite them in terms of Legendre polynomials as follows:

$$
\begin{aligned}
P u_{-} & =\sum_{k=0}^{N-2}\left(\hat{u}_{\phi}\right)_{k} L_{k}+c_{N-1} L_{N-1}+c_{N} L_{N}, \\
P u_{+} & =\sum_{k=0}^{N-2}\left(\hat{u}_{\psi}\right)_{k} L_{k}+d_{N-1} L_{N-1}+d_{N} L_{N},
\end{aligned}
$$


where

$$
\begin{aligned}
c_{N-1}= & \sum_{k=0}^{\frac{N-4}{2}}\left[-\beta_{2 k+1}\left(\hat{u}_{\phi}\right)_{2 k+1}+\gamma_{2 k+1}\left(\hat{u}_{\psi}\right)_{2 k+1}\right], \\
c_{N}= & \sum_{k=1}^{\frac{N-2}{2}}\left[-\beta_{2 k}\left(\hat{u}_{\phi}\right)_{2 k}+\gamma_{2 k}\left(\hat{u}_{\psi}\right)_{2 k}\right], \\
d_{N-1}= & \sum_{k=0}^{\frac{N-4}{2}}\left[\gamma_{2 k+1}\left(\hat{u}_{\phi}\right)_{2 k+1}-\beta_{2 k+1}\left(\hat{u}_{\psi}\right)_{2 k+1}\right], \\
d_{N}= & \sum_{k=1}^{\frac{N-2}{2}}\left[\gamma_{2 k}\left(\hat{u}_{\phi}\right)_{2 k}-\beta_{2 k}\left(\hat{u}_{\psi}\right)_{2 k}\right] .
\end{aligned}
$$

Now we identify

$$
\begin{aligned}
\left(\hat{u}_{\phi}\right)_{k} & =\frac{2 k+1}{2} \int_{-1}^{1} u_{-}\left(x_{-}(\xi)\right) L_{k}(\xi) d \xi \\
\left(\hat{u}_{\psi}\right)_{k} & =\frac{2 k+1}{2} \int_{-1}^{1} u_{+}\left(x_{+}(\xi)\right) L_{k}(\xi) d \xi
\end{aligned}
$$

which are exactly the Legendre coefficients for $u_{-}$and $u_{+}$, respectively. For clarity, we replace the notation $\left(\hat{u}_{\phi}\right)_{k}$ by $\left(\hat{u}_{-}\right)_{k}$, and similarly $\left(\hat{u}_{\psi}\right)_{k}$ by $\left(\hat{u}_{+}\right)_{k}$. Then, considering an eigenfunction $u_{i}$, the expansion coefficients of $P u_{i}$ are denoted by $\left(\hat{u}_{i_{-}}\right)_{k}$ and $\left(\hat{u}_{i_{+}}\right)_{k}$. Then we have

$$
\begin{aligned}
& \left|\left(u_{i}-P u_{i}, u_{j}\right)_{-}\right| \\
& =\left|\left(\sum_{k \geq N-1}^{\infty}\left(\hat{u}_{i_{-}}\right)_{k} L_{k}-c_{N-1} L_{N-1}-c_{N} L_{N}, \sum_{n=0}^{\infty}\left(\hat{u}_{j_{-}}\right)_{n} L_{n}\right)\right| \\
& \leq \frac{\pi}{2} \sum_{k \geq N-1}^{\infty}\left|\left(\hat{u}_{i_{-}}\right)_{k}\right|\left|\left(\hat{u}_{j_{-}}\right)_{k}\right| \int_{-1}^{1} L_{k}^{2} d \xi \\
& +\frac{\pi}{2}\left|c_{N-1}\right|\left|\left(\hat{u}_{j_{-}}\right)_{N-1}\right| \int_{-1}^{1} L_{N-1}^{2} d \xi+\frac{\pi}{2}\left|c_{N}\right|\left|\left(\hat{u}_{j_{-}}\right)_{N}\right| \int_{-1}^{1} L_{N}^{2} d \xi=R H S(1) .
\end{aligned}
$$

We examine the two terms $\left|c_{N-1}\right|$ and $\left|c_{N}\right|$. From the Cauchy-Schwarz inequality, we have

$$
\begin{aligned}
\left|c_{N-1}\right| & \leq\left\{\sum_{k=0}^{\frac{N-4}{2}}\left(\left|\beta_{2 k+1}\right|^{2}+\left|\gamma_{2 k+1}\right|^{2}\right)\right\}^{1 / 2}\left\{\sum_{k=0}^{\frac{N-4}{2}}\left(\left|\left(\hat{u}_{i_{-}}\right)_{2 k+1}\right|^{2}+\left|\left(\hat{u}_{i_{+}}\right)_{2 k+1}\right|^{2}\right)\right\}^{1 / 2}, \\
\left|c_{N}\right| & \leq\left\{\sum_{k=1}^{\frac{N-2}{2}}\left(\left|\beta_{2 k}\right|^{2}+\left|\gamma_{2 k}\right|^{2}\right)\right\}^{1 / 2}\left\{\sum_{k=1}^{\frac{N-2}{2}}\left(\left|\left(\hat{u}_{i_{-}}\right)_{2 k}\right|^{2}+\left|\left(\hat{u}_{i_{+}}\right)_{2 k}\right|^{2}\right)\right\}^{1 / 2} .
\end{aligned}
$$

Since $\left|\beta_{k}\right|,\left|\gamma_{k}\right|<1$, and $\left|\left(\hat{u}_{j_{-}}\right)_{k}\right|,\left|\left(\hat{u}_{j_{+}}\right)_{k}\right|$ decay like $O\left(k^{-m}\right)$ in [4] and [5], it is clear that $\left|c_{N-1}\right|$ and $\left|c_{N}\right|$ are bounded by $O(N)$. Since $\int_{-1}^{1} L_{k}^{2}(\xi) d \xi=\frac{2}{2 k+1}$, the second 
term of $R H S(1)$ which is the leading term decays like $O\left(N^{-m}\right)$. Therefore we have

$$
\left|\left(u_{i}-P u_{i}, u_{j}\right)_{-}\right| \leq C N^{-m} .
$$

Similarly, we get

$$
\left|\left(u_{i}-P u_{i}, u_{j}\right)_{+}\right| \leq C N^{-m} .
$$

This completes the proof.

LEMma 4.3. For $u_{i=1, \ldots, l} \in H_{p}^{1}(\Omega)$, where $\left(u_{i}\right)_{-} \in H^{m}\left(\Omega_{-}\right)$and $\left(u_{i}\right)_{+} \in$ $H^{m}\left(\Omega_{+}\right)$,

$$
\max _{v \in E 1, l} \frac{a(P v, P v)}{a(v, v)} \leq 1+C(l) N^{-m},
$$

where the constant $C(l)$ is independent of $N$.

Proof. Since

$$
\frac{a(P v, P v)}{a(v, v)}=1-\frac{a(v, v)-a(P v, P v)}{a(v, v)}
$$

we examine the convergency of the last term (4.21). Following the similar procedure as in Lemma 4.2, we obtain

$$
\begin{aligned}
\left|a\left(u_{i}-P u_{i}, u_{j}\right)_{-}\right|= & \left|a\left(\sum_{k \geq N-1}^{\infty}\left(\hat{u}_{i_{-}}\right)_{k} L_{k}-c_{N-1} L_{N-1}-c_{N} L_{N}, \sum_{n=0}^{\infty}\left(\hat{u}_{j_{-}}\right)_{n} L_{n}\right)\right| \\
& \leq \frac{\pi}{2} \sum_{k \geq N-1}^{\infty} \sum_{n=0}^{\infty}\left|\left(\hat{u}_{i_{-}}\right)_{k}\right|\left|\left(\hat{u}_{j_{-}}\right)_{k}\right| \int_{-1}^{1} L_{k}^{\prime} L_{n}^{\prime} d \xi \\
& +\frac{\pi}{2}\left|c_{N-1}\right|\left|\left(\hat{u}_{j_{-}}\right)_{N-1}\right| \sum_{n=0}^{\infty} \int_{-1}^{1} L_{N-1}^{\prime} L_{n}^{\prime} d \xi \\
& +\frac{\pi}{2}\left|c_{N}\right|\left|\left(\hat{u}_{j_{-}}\right)_{N}\right| \sum_{n=0}^{\infty} \int_{-1}^{1} L_{N}^{\prime} L_{n}^{\prime} d \xi=\operatorname{RHS}(2) .
\end{aligned}
$$

Since the leading term of $R H S(2)$ decays like $O\left(N^{-m}\right)$, we have

$$
\left|a\left(u_{i}-P u_{i}, u_{j}\right)_{-}\right| \leq C N^{-m} .
$$

Similarly, we get

$$
\left|a\left(u_{i}-P u_{i}, u_{j}\right)_{+}\right| \leq C N^{-m} .
$$

This completes the proof.

As consequences of Lemmas 4.2 and 4.3, we have the following theorems.

THEOREM 4.4. For $u_{i=1, \ldots, l} \in H_{p}^{1}(\Omega)$, where $\left(u_{i}\right)_{-} \in H^{m}\left(\Omega_{-}\right)$and $\left(u_{i}\right)_{+} \in$ $H^{m}\left(\Omega_{+}\right)$, let $\lambda_{l}^{N}$ be the lth eigenvalue obtained by the multidomain Legendre-Galerkin approximation from (4.1) to the eigenvalue $\lambda_{l}$. Then

$$
\left|\lambda_{l}^{N}-\lambda_{l}\right| \leq C(l) N^{-m},
$$

where $C(l)$ is independent of $N$. 
For the approximate eigenvectors, we can state the following.

THEOREM 4.5. For $u_{i=1, \ldots, l} \in H_{p}^{1}(\Omega)$, where $\left(u_{i}\right)_{-} \in H^{m}\left(\Omega_{-}\right)$and $\left(u_{i}\right)_{+} \in$ $H^{m}\left(\Omega_{+}\right)$, let $u_{l}^{N}$ be the lth eigenfunction of the multidomain Legendre-Galerkin approximation (4.1) to the eigenfunction $u_{l}$. Then

$$
\left\|u_{l}-u_{l}^{N}\right\| \leq C(l) N^{-m}
$$

where $C(l)$ is independent of $N$.

The proof follows the same way as in [21].

5. Legendre-collocation methods. The Legendre-Gauss-Lobatto points $\xi_{i}$ are defined by

$$
\xi_{0}=-1, \quad \xi_{N}=1, \quad \xi_{i}(i=1, \ldots, N-1) \text { zeros of } L_{N}^{\prime},
$$

and the Legendre-Gauss-Lobatto weights are

$$
w_{i}=\frac{2}{N(N+1)} \frac{1}{\left[L_{N}\left(\xi_{i}\right)\right]^{2}} .
$$

Denoting

$$
\begin{array}{rlrl}
\left(u_{-}\right)_{i} & =\left.u\right|_{\left(x_{-}\right)_{i}} & \text { for }\left(x_{-}\right)_{i} & =\frac{\pi}{2}\left(\xi_{i}-1\right), \\
\left(u_{+}\right)_{i}=\left.u\right|_{\left(x_{+}\right)_{i}} & \text { for }\left(x_{+}\right)_{i}=\frac{\pi}{2}\left(\xi_{i}+1\right),
\end{array}
$$

we define two discrete bilinear forms that approximate $a(u, v)_{-}$and $a(u, v)_{+}$:

$$
\begin{aligned}
& a(u, v)_{h-}=\sum_{i=0}^{N}\left(u_{-}\right)_{i}^{\prime}\left(v_{-}\right)_{i}^{\prime} w_{i}+\left(u_{-}\right)_{0}^{\prime}\left(v_{-}\right)_{0}-\left(u_{-}\right)_{N}^{\prime}\left(v_{-}\right)_{N}, \\
& a(u, v)_{h+}=\sum_{i=0}^{N}\left(u_{+}\right)_{i}^{\prime}\left(v_{+}\right)_{i}^{\prime} w_{i}+\left(u_{+}\right)_{0}^{\prime}\left(v_{+}\right)_{0}-\left(u_{+}\right)_{N}^{\prime}\left(v_{+}\right)_{N} .
\end{aligned}
$$

To approximate $(u, v)_{-}$and $(u, v)_{+}$, define

$$
\begin{aligned}
& (u, v)_{h-}=\sum_{i=0}^{N}\left(u_{-}\right)_{i}\left(v_{-}\right)_{i}\left(\epsilon_{-}\right)_{i} w_{i} \\
& (u, v)_{h+}=\sum_{i=0}^{N}\left(u_{+}\right)_{i}\left(v_{+}\right)_{i}\left(\epsilon_{+}\right)_{i} w_{i} .
\end{aligned}
$$

It is natural to define the following discrete bilinear forms approximating the continuous bilinear forms $a(u, v)$ and $(u, v)$ :

$$
\begin{aligned}
a(u, v)_{h} & =a(u, v)_{h-}+a(u, v)_{h+}, \\
(u, v)_{h} & =(u, v)_{h-}+(u, v)_{h+} .
\end{aligned}
$$

Now, we state the multidomain discrete variational formulation of (2.2): Find $\lambda^{c}$ and $u^{c}$ in $V_{2 N-2}$ such that

$$
a\left(u^{c}, v\right)_{h}=\lambda^{c}\left(u^{c}, v\right)_{h} \quad \text { for } v \in V,
$$

where $V$ is a suitable space that will be specified later. In the following subsections, we introduce two different Legendre-collocation methods by taking the space $V$ differently. 
5.1. Legendre-collocation method 1 . Our first Legendre-collocation method takes the space $V_{2 N-2}$ as a test space. Find $\lambda^{c}$ and $u^{c}$ in $V_{2 N-2}$ such that

$$
a\left(u^{c}, v^{c}\right)_{h}=\lambda^{c}\left(u^{c}, v^{c}\right)_{h} \text { for } v^{c} \in V_{2 N-2} .
$$

To construct the scheme, we expand

$$
\begin{aligned}
& \left(u_{-}^{c}\right)_{i}=\sum_{j=0}^{N}\left(u_{-}^{c}\right)_{j} l_{j}\left(\xi_{i}\right), \\
& \left(u_{+}^{c}\right)_{i}=\sum_{j=0}^{N}\left(u_{+}^{c}\right)_{j} l_{j}\left(\xi_{i}\right),
\end{aligned}
$$

where the Lagrange interpolation polynomials of degree $N$ based on the LegendreGauss-Lobatto points [8], [10] are

$$
l_{j}(\xi)=-\frac{1}{N(N+1)} \frac{\left(1-\xi^{2}\right) L_{N}^{\prime}(\xi)}{\left(\xi-\xi_{j}\right) L_{N}\left(\xi_{j}\right)} .
$$

Take $v^{c}=\phi_{n}(x)(0 \leq n \leq N-2)$, which is the basis for $V_{2 N-2}$, and substitute $u_{-}^{c}$, $u_{+}^{c}$, and $v^{c}$ in (5.5) and (5.6). Applying the continuity and the periodicity for $u^{c}$, that is, $\left(u_{-}^{c}\right)_{0}=\left(u_{+}^{c}\right)_{N}$ and $\left(u_{-}^{c}\right)_{N}=\left(u_{+}^{c}\right)_{0}$, we get

$$
\begin{aligned}
a\left(u^{c}, v^{c}\right)_{h} & =\left(u_{-}^{c}\right)_{0}\left(\sum_{i=0}^{N}\left[D_{i 0}\left(\phi_{n_{-}}\right)_{i}^{\prime} w_{0}+D_{i N}\left(\phi_{n_{-}}\right)_{i}^{\prime} w_{N}\right]\right) \\
& +\left(u_{+}^{c}\right)_{0}\left(\sum_{i=0}^{N}\left[D_{i 0}\left(\phi_{n_{+}}\right)_{i}^{\prime} w_{0}+D_{i N}\left(\phi_{n_{+}}\right)_{i}^{\prime} w_{N}\right]\right) \\
& +\sum_{j=1}^{N-1}\left(u_{-}^{c}\right)_{j} \sum_{i=0}^{N} D_{i j}\left(\phi_{n_{-}}\right)_{i}^{\prime} w_{i}+\sum_{j=1}^{N-1}\left(u_{+}^{c}\right)_{j} \sum_{i=0}^{N} D_{i j}\left(\phi_{n_{+}}\right)_{i}^{\prime} w_{i},
\end{aligned}
$$

where $D_{i j}=l_{j}^{\prime}\left(\xi_{i}\right)$ is the differentiation matrix of Lagrange polynomials based on Legendre-Gauss-Lobatto points [10], [13]. Similarly, we have

$$
\begin{aligned}
\left(u^{c}, v^{c}\right)_{h} & =\left(u_{-}^{c}\right)_{0}\left[\left(\phi_{0_{-}}\right)_{0}\left(\epsilon_{-}\right)_{0} w_{0}+\left(\phi_{0_{+}}\right)_{N}\left(\epsilon_{+}\right)_{N} w_{N}\right] \\
& +\left(u_{+}^{c}\right)_{0}\left[\left(\phi_{0_{+}}\right)_{0}\left(\epsilon_{+}\right)_{0} w_{0}+\left(\phi_{0_{-}}\right)_{N}\left(\epsilon_{-}\right)_{N} w_{N}\right] \\
& +\sum_{j=1}^{N-1}\left(u_{-}^{c}\right)_{j}\left(\phi_{n_{-}}\right)_{j}\left(\epsilon_{-}\right)_{j} w_{j}+\sum_{j=1}^{N-1}\left(u_{+}^{c}\right)_{j}\left(\phi_{n_{+}}\right)_{j}\left(\epsilon_{+}\right)_{j} w_{j} .
\end{aligned}
$$

Applying the same procedure for $v^{c}=\psi_{n}(x)(0 \leq n \leq N-2)$, we have a system of $2 N-2$ equations with the unknown vector

$$
\mathbf{u}^{c}=\left[\left(u_{-}^{c}\right)_{0},\left(u_{-}^{c}\right)_{1}, \ldots,\left(u_{-}^{c}\right)_{N-1},\left(u_{+}^{c}\right)_{0},\left(u_{+}^{c}\right)_{1}, \ldots,\left(u_{+}^{c}\right)_{N-1}\right]^{T} .
$$

From the remaining boundary and the interface conditions, that is, $\left(u_{-}^{c}\right)_{0}^{\prime}=\left(u_{+}^{c}\right)_{N}^{\prime}$ and $\left(u_{-}^{c}\right)_{N}^{\prime}=\left(u_{+}^{c}\right)_{0}^{\prime}$, we get two more equations:

$$
\begin{aligned}
& \left(u_{-}^{c}\right)_{0}\left[D_{0 N}-D_{N 0}\right]+\sum_{j=1}^{N-1}\left(u_{-}^{c}\right)_{j} D_{j N}+\left(u_{+}^{c}\right)_{0}\left[D_{N N}-D_{00}\right]-\sum_{j=1}^{N-1}\left(u_{+}^{c}\right)_{j} D_{j 0}=0, \\
& \left(u_{-}^{c}\right)_{0}\left[D_{0 N}-D_{N 0}\right]+\sum_{j=1}^{N-1}\left(u_{-}^{c}\right)_{j} D_{j 0}+\left(u_{+}^{c}\right)_{0}\left[D_{N N}-D_{00}\right]-\sum_{j=1}^{N-1}\left(u_{+}^{c}\right)_{j} D_{j N}=0 .
\end{aligned}
$$


Finally, we can represent the Legendre-collocation (method 1) scheme (5.11) in matrix form:

$$
K \mathbf{u}^{c}=\lambda^{c} M \mathbf{u}^{c},
$$

where the dimension of the matrices $K$ and $M$ is $2 N \times 2 N$.

The numerical results are presented in Table 2 for the first few eigenvalues in ascending order and the corresponding eigenfunctions, showing that the relative errors for eigenvalues and $l_{2}$-errors of the eigenfunctions decay exponentially as $N$ increases.

5.2. Legendre-collocation method 2. Let us first define the $(N-1)$-dimensional space

$$
\overline{\mathcal{L}}_{N-1}=\operatorname{span}\left\{l_{j}(\xi) \mid 1 \leq j \leq N-1, \quad \xi \in[-1,1]\right\},
$$

where the Lagrange interpolation polynomials of degree $N$ based on the LegendreGauss-Lobatto points are

$$
l_{j}(\xi)=-\frac{1}{N(N+1)} \frac{\left(1-\xi^{2}\right) L_{N}^{\prime}(\xi)}{\left(\xi-\xi_{j}\right) L_{N}\left(\xi_{j}\right)} .
$$

Then we define

$$
W_{2 N-2}=\left\{\varphi \in C_{p}^{0}(\Omega) \mid \varphi_{-}\left(x_{-}(\xi)\right) \in \overline{\mathcal{L}}_{N-1} \text { and } \varphi_{+}\left(x_{+}(\xi)\right) \in \overline{\mathcal{L}}_{N-1}\right\},
$$

the basis of which is given by $\left\{\varphi_{n}, \zeta_{n}\right\}_{n=1}^{N-1}$, where

$$
\varphi_{n}=\left\{\begin{array}{c}
l_{n}(\xi) \text { in }[-\pi, 0], \\
0 \text { otherwise }
\end{array} \text { and } \zeta_{n}=\left\{\begin{array}{cc}
0 & \text { in }[-\pi, 0], \\
l_{n}(\xi) & \text { otherwise }
\end{array}\right.\right.
$$

The multidomain discrete variational formulation of (2.2) for this method is to find $\lambda^{c c}$ and $u^{c c}$ in $V_{2 N-2}$ such that

$$
a\left(u^{c c}, w^{c}\right)_{h}=\lambda^{c c}\left(u^{c c}, w^{c}\right)_{h} \text { for } w^{c} \in W_{2 N-2} .
$$

Setting

$$
\begin{aligned}
\left(u_{-}^{c c}\right)_{i} & =\sum_{j=0}^{N}\left(u_{-}^{c c}\right)_{j} l_{j}\left(\xi_{i}\right), \\
\left(u_{+}^{c c}\right)_{i} & =\sum_{j=0}^{N}\left(u_{+}^{c c}\right)_{j} l_{j}\left(\xi_{i}\right)
\end{aligned}
$$

and plugging them into (5.20) with $w^{c}=\varphi_{n}(x), \zeta_{n}(x)(1 \leq n \leq N-1)$, we get

$$
K \mathbf{u}^{c c}=\lambda^{c c} M \mathbf{u}^{c c},
$$

where, denoting the second derivative matrix of Lagrange interpolation polynomials of degree $N$ based on Legendre-Gauss-Lobatto points by $D^{2}$, the entries of $(2 N \times 2 N)$ matrices $K$ and $M$ are expressed by

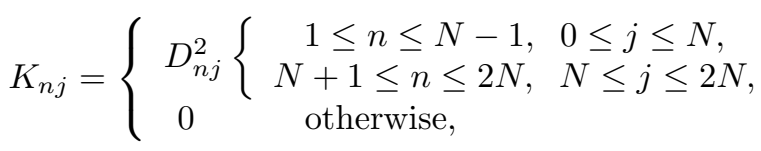


TABLE 2

Domain decomposition Legendre-collocation methods ( $L C 1=$ method $1, L C 2=$ Method 2 ) for the relative errors of eigenvalues for $\omega=2$ and the $l_{2}$-discrete errors of eigenfunctions.

\begin{tabular}{|c||c||c|c|c||c|}
\hline \multicolumn{2}{|c|}{ Methods } & \multicolumn{2}{c|}{ LC1 } & \multicolumn{2}{c|}{ LC2 } \\
\hline$\lambda_{i}$ & $N$ & $\left(\lambda_{i}^{c}-\lambda_{i}\right) / \lambda_{i}$ & $\left\|u_{i}-u_{i}^{c}\right\|$ & $\left(\lambda_{i}^{c c}-\lambda_{i}\right) / \lambda_{i}$ & $\left\|u_{i}-u_{i}^{c c}\right\|$ \\
\hline \multirow{5}{*}{0.369875} & 4 & $2.94(-03)$ & $2.91(-03)$ & $3.81(-01)$ & $1.97(-01)$ \\
& 8 & $7.04(-10)$ & $4.65(-07)$ & $-5.74(-05)$ & $2.95(-06)$ \\
& 16 & $-1.75(-14)$ & $2.33(-15)$ & $-2.61(-13)$ & $1.35(-14)$ \\
& 32 & $-2.23(-13)$ & $3.19(-14)$ & $2.30(-12)$ & $1.40(-13)$ \\
& 64 & $-6.66(-13)$ & $3.32(-13)$ & $9.42(-12)$ & $4.81(-13)$ \\
\hline & 4 & $-6.14(-03)$ & $2.17(-02)$ & $1.39(+00)$ & $1.06(+00)$ \\
& 8 & $-8.61(-09)$ & $4.22(-06)$ & $-3.38(-05)$ & $2.99(-05)$ \\
0.536233 & 16 & $-2.48(-15)$ & $3.10(-14)$ & $-1.49(-13)$ & $3.10(-13)$ \\
& 32 & $0.00(+00)$ & $1.59(-13)$ & $5.81(-14)$ & $4.88(-13)$ \\
& 64 & $-2.05(-13)$ & $2.46(-13)$ & $-5.60(-13)$ & $2.18(-12)$ \\
\hline \multirow{5}{*}{1.607115} & 4 & $-7.80(-02)$ & $1.29(-01)$ & $8.07(-01)$ & $8.79(-01)$ \\
& 8 & $-2.89(-05)$ & $6.33(-04)$ & $-2.10(-03)$ & $5.43(-03)$ \\
& 16 & $6.90(-16)$ & $7.33(-11)$ & $-8.33(-10)$ & $1.59(-09)$ \\
& 32 & $1.28(-14)$ & $2.26(-13)$ & $2.99(-14)$ & $4.62(-13)$ \\
& 64 & $-4.42(-15)$ & $6.55(-13)$ & $-1.85(-13)$ & $1.35(-12)$ \\
\hline \multirow{5}{*}{1.937181} & 4 & $3.28(-01)$ & $1.17(-01)$ & $1.62(+00)$ & $2.49(-01)$ \\
& 8 & $1.45(-04)$ & $4.99(-04)$ & $1.13(-02)$ & $1.89(-03)$ \\
& 16 & $1.83(-15)$ & $2.38(-10)$ & $2.20(-08)$ & $3.83(-09)$ \\
& 32 & $-6.49(-14)$ & $3.86(-14)$ & $4.56(-13)$ & $8.42(-14)$ \\
& 64 & $-2.68(-13)$ & $1.24(-13)$ & $1.85(-12)$ & $3.32(-13)$ \\
\hline
\end{tabular}

and

$$
M_{n j}=\operatorname{diag}\left\{\left(\epsilon_{-}\right)_{1}, \ldots,\left(\epsilon_{-}\right)_{N-1},\left(\epsilon_{+}\right)_{1}, \ldots,\left(\epsilon_{+}\right)_{N-1}\right\} .
$$

Additionally, two more equations are incorporated into the first and $N$ th rows of the matrices $K$ and $M$, which are from the boundary and interface constraints:

$$
\begin{aligned}
& \left(u_{-}^{c c}\right)_{0}\left[D_{0 N}-D_{N 0}\right]+\sum_{j=1}^{N-1}\left(u_{-}^{c c}\right)_{j} D_{j N}+\left(u_{+}^{c c}\right)_{0}\left[D_{N N}-D_{00}\right]-\sum_{j=1}^{N-1}\left(u_{+}^{c c}\right)_{j} D_{j 0}=0, \\
& \left(u_{-}^{c c}\right)_{0}\left[D_{0 N}-D_{N 0}\right]+\sum_{j=1}^{N-1}\left(u_{-}^{c c}\right)_{j} D_{j 0}+\left(u_{+}^{c c}\right)_{0}\left[D_{N N}-D_{00}\right]-\sum_{j=1}^{N-1}\left(u_{+}^{c c}\right)_{j} D_{j N}=0
\end{aligned}
$$

where $D$ is defined as in section 5.1.

Solving (5.21) numerically, one obtains the eigenvalues $\lambda_{l}^{c c}(l \leq 2 N)$ and the associated eigenvector

$$
\mathbf{u}^{c c}=\left[\left(u_{-}^{c c}\right)_{0},\left(u_{-}^{c c}\right)_{1}, \ldots,\left(u_{-}^{c c}\right)_{N-1},\left(u_{+}^{c c}\right)_{0},\left(u_{+}^{c c}\right)_{1}, \ldots,\left(u_{+}^{c c}\right)_{N-1}\right]^{T} .
$$

The numerical results are presented in Table 2 for the first few eigenvalues in ascending order and the corresponding eigenfunctions, showing the exponential rate of convergence.

6. Legendre-collocation penalty method. In this section, the notation used in section 4 represents the same definition. Let $\mathcal{L}_{N+1}$ be the $(N+1)$-dimensional space of Legendre-Lagrange interpolation polynomials of degree $N$ defined by

$$
\mathcal{L}_{N+1}=\operatorname{span}\left\{l_{j}(\xi) \mid 0 \leq j \leq N, \quad \xi \in[-1,1]\right\},
$$


TABLE 3

Relative errors of eigenvalues $\omega=2$ and the $l_{2}$-discrete errors of eigenfunctions for multidomain Chebyshev-collocation method 2.

\begin{tabular}{|c||c||c|c|}
\hline$\lambda_{i}$ & $N$ & $\left(\lambda_{i}^{c c}-\lambda_{i}\right) / \lambda_{i}$ & $\left\|u_{i}-u_{i}^{c c}\right\|$ \\
\hline & 4 & $-2.75(-02)$ & $2.81(-03)$ \\
& 8 & $-2.20(-05)$ & $1.22(-06)$ \\
0.369875 & 16 & $8.55(-15)$ & $1.99(-15)$ \\
& 32 & $-1.76(-13)$ & $2.80(-14)$ \\
& 64 & $-4.58(-12)$ & $1.15(-12)$ \\
\hline \multirow{5}{*}{0.536233} & 4 & $4.21(-02)$ & $2.51(-02)$ \\
& 8 & $-1.39(-05)$ & $1.18(-05)$ \\
& 16 & $-5.77(-14)$ & $5.06(-14)$ \\
& 32 & $-5.38(-14)$ & $1.72(-13)$ \\
& 64 & $-3.55(-13)$ & $9.13(-13)$ \\
\hline \multirow{5}{*}{1.607115} & 4 & $1.55(-01)$ & $4.70(-01)$ \\
& 8 & $-8.48(-04)$ & $1.82(-03)$ \\
& 16 & $-2.28(-10)$ & $4.31(-10)$ \\
& 32 & $4.98(-14)$ & $8.25(-14)$ \\
& 64 & $-1.41(-13)$ & $9.66(-13)$ \\
\hline & 4 & $6.97(-01)$ & $4.74(-02)$ \\
& 8 & $4.52(-03)$ & $6.72(-04)$ \\
& 16 & $6.13(-09)$ & $1.05(-09)$ \\
& 32 & $-2.88(-14)$ & $1.56(-14)$ \\
& 64 & $-8.99(-13)$ & $4.72(-13)$ \\
\hline
\end{tabular}

where

$$
l_{j}(\xi)=-\frac{1}{N(N+1)} \frac{\left(1-\xi^{2}\right) L_{N}^{\prime}(\xi)}{\left(\xi-\xi_{j}\right) L_{N}\left(\xi_{j}\right)} .
$$

Let $Y_{2 N+2}$ be the $(2 N+2)$-dimensional space of piecewise continuous interpolation polynomials defined as

$$
Y_{2 N+2}=\left\{\eta \in L^{2}(\Omega) \mid \eta_{-}\left(x_{-}(\xi)\right) \in \mathcal{L}_{N+1} \text { and } \eta_{+}\left(x_{+}(\xi)\right) \in \mathcal{L}_{N+1}\right\},
$$

the basis of which is given by

$$
\left\{\eta_{n}, \varsigma_{n}\right\} \quad \text { for } 0 \leq n \leq N
$$

where

$$
\eta_{n}=\left\{\begin{array}{c}
l_{n}(\xi) \text { in }[-\pi, 0], \\
0 \text { otherwise }
\end{array} \quad \text { and } \quad \varsigma_{n}=\left\{\begin{array}{c}
0 \text { in }(-\pi, 0) \\
l_{n}(\xi) \text { otherwise }
\end{array}\right.\right.
$$

Now, we define the discrete bilinear form approximating $a(u, v)$ with penalty boundary constraints:

$$
\begin{aligned}
a(u, v)_{\tau}= & a(u, v)_{h_{-}}+a(u, v)_{h_{+}} \\
& +\tau_{1}\left\{\left(u_{+}\right)_{N}-\left(u_{-}\right)_{0}\right\} \\
& +\tau_{2}\left\{\left(u_{+}\right)_{0}-\left(u_{-}\right)_{N}\right\} \\
& +\tau_{3}\left\{\left(u_{+}\right)_{N}^{\prime}-\left(u_{-}\right)_{0}^{\prime}\right\} \\
& +\tau_{4}\left\{\left(u_{-}\right)_{N}^{\prime}-\left(u_{+}\right)_{0}^{\prime}\right\}
\end{aligned}
$$


where $\tau_{i}(1 \leq i \leq 4)$ are suitable constants depending on $N$, to be chosen later.

Now, we state the multidomain discrete variational formulation of (2.2) by penalty approach. Find $\lambda^{\tau}$ and $u^{\tau}$ in $Y_{2 N+2}$ such that

$$
a\left(u^{\tau}, v^{\tau}\right)_{\tau}=\lambda^{\tau}\left(u^{\tau}, v^{\tau}\right)_{h} \text { for } v^{\tau} \in Y_{2 N+2} .
$$

To construct the scheme, we expand

$$
\begin{aligned}
& \left(u_{-}^{\tau}\right)_{i}=\sum_{j=0}^{N}\left(u_{-}^{\tau}\right)_{j} l_{j}\left(\xi_{i}\right), \\
& \left(u_{+}^{\tau}\right)_{i}=\sum_{j=0}^{N}\left(u_{+}^{\tau}\right)_{j} l_{j}\left(\xi_{i}\right) .
\end{aligned}
$$

Take $v^{\tau}=\eta_{n}(x)$ and $v^{\tau}=\varsigma_{n}(x)$, and choose $\tau_{1}=\tau_{2}=\sigma_{1}^{\tau}$ and $\tau_{3}=\tau_{4}=\sigma_{2}^{\tau}$. Then we define the following matrix $K$, whose dimensions are $2 N+2$ :

$$
K=\left[\begin{array}{cc}
D^{2} & O \\
O & D^{2}
\end{array}\right],
$$

where the $(N+1) \times(N+1)$ matrix $D^{2}$ is the second derivative matrix of Lagrange interpolation polynomials based on Legendre-Gauss-Lobatto points. Also, defining the matrices

$$
\begin{gathered}
M=\operatorname{diag}\left\{\left(\epsilon_{-}\right)_{0}, \ldots,\left(\epsilon_{-}\right)_{N},\left(\epsilon_{+}\right)_{0}, \ldots,\left(\epsilon_{+}\right)_{N}\right\}, \\
B_{1}=\left[\begin{array}{cccccc}
-1 & \ldots & 0 & 0 & \ldots & 1 \\
0 & \ldots & 0 & 0 & \ldots & 0 \\
\vdots & \ldots & 0 & 0 & \ldots & \vdots \\
0 & \ldots & -1 & 1 & \ldots & 0 \\
0 & \ldots & -1 & 1 & \ldots & 0 \\
\vdots & \ldots & 0 & 0 & \ldots & \vdots \\
0 & \ldots & 0 & 0 & \ldots & 0 \\
-1 & \ldots & 0 & 0 & \ldots & 1
\end{array}\right]
\end{gathered}
$$

and

$$
B_{2}=\left[\begin{array}{cccccc}
-D_{01} & \ldots & -D_{0 N} & D_{N 1} & \ldots & D_{N N} \\
0 & \ldots & 0 & 0 & \ldots & 0 \\
\vdots & \ldots & \vdots & \vdots & \ldots & \vdots \\
0 & \ldots & 0 & 0 & \ldots & 0 \\
D_{N 1} & \ldots & D_{N N} & -D_{01} & \ldots & -D_{0 N} \\
-D_{01} & \ldots & -D_{0 N} & D_{N 1} & \ldots & D_{N N} \\
0 & \ldots & 0 & 0 & \ldots & 0 \\
\vdots & \ldots & \vdots & \vdots & \ldots & \vdots \\
0 & \ldots & 0 & 0 & \ldots & 0 \\
D_{N 1} & \ldots & D_{N N} & -D_{01} & \ldots & -D_{0 N}
\end{array}\right]
$$

and letting

$$
K_{\tau}=K+\sigma_{1}^{\tau} B_{1}+\sigma_{2}^{\tau} B_{2},
$$


TABLE 4

Relative errors of eigenvalues for $\omega=2$ and the $L_{2}$-discrete errors of eigenfunctions for the Legendre-collocation penalty (LCP) method and the Chebyshev-collocation penalty (CCP) method.

\begin{tabular}{|c||c||c|c||c|c|}
\hline \multicolumn{2}{|c|}{ Methods } & \multicolumn{2}{c|}{ LCP } & \multicolumn{2}{c|}{ CCP } \\
\hline$\lambda_{i}$ & $N$ & $\left(\lambda_{i}^{\tau}-\lambda_{i}\right) / \lambda_{i}$ & $\left\|u_{i}-u_{i}^{\tau}\right\|$ & $\left(\lambda_{i}^{\tau}-\lambda_{i}\right) / \lambda_{i}$ & $\left\|u_{i}-u_{i}^{\tau}\right\|$ \\
\hline \multirow{5}{*}{0.369875} & 4 & $-6.35(-02)$ & $2.91(-03)$ & $-2.76(-02)$ & $2.64(-03)$ \\
& 8 & $-5.73(-05)$ & $5.09(-06)$ & $-2.21(-05)$ & $1.18(-06)$ \\
& 16 & $-2.18(-13)$ & $2.53(-14)$ & $-1.79(-12)$ & $4.17(-13)$ \\
& 32 & $5.10(-12)$ & $4.75(-13)$ & $-5.86(-12)$ & $1.37(-12)$ \\
& 64 & $-8.03(-12)$ & $3.88(-12)$ & $-1.28(-10)$ & $2.83(-11)$ \\
\hline & 4 & $-4.81(-02)$ & $4.12(-02)$ & $-4.30(-02)$ & $2.43(-02)$ \\
& 8 & $-3.40(-05)$ & $4.01(-05)$ & $-1.41(-05)$ & $1.13(-05)$ \\
0.536233 & 16 & $-6.40(-13)$ & $3.75(-12)$ & $4.47(-13)$ & $3.07(-12)$ \\
& 32 & $2.05(-13)$ & $1.72(-12)$ & $2.44(-12)$ & $1.73(-11)$ \\
& 64 & $3.90(-11)$ & $3.47(-10)$ & $4.10(-11)$ & $2.93(-10)$ \\
\hline & 4 & $-1.96(-01)$ & $4.42(-01)$ & $-1.52(-01)$ & $4.53(-01)$ \\
& 8 & $-2.10(-03)$ & $4.06(-03)$ & $-8.34(-04)$ & $1.70(-03)$ \\
& 16 & $-8.32(-10)$ & $1.50(-09)$ & $-2.26(-10)$ & $4.18(-10)$ \\
& 32 & $1.70(-12)$ & $4.20(-11)$ & $6.39(-13)$ & $6.93(-12)$ \\
& 64 & $-2.21(-12)$ & $8.62(-11)$ & $1.20(-11)$ & $1.36(-10)$ \\
\hline \multirow{5}{*}{1.607115} & 4 & $5.11(-01)$ & $5.28(-02)$ & $7.13(-01)$ & $5.81(-02)$ \\
& 8 & $1.13(-02)$ & $1.82(-03)$ & $4.57(-03)$ & $6.38(-04)$ \\
& 16 & $2.20(-08)$ & $3.71(-09)$ & $6.15(-09)$ & $1.02(-09)$ \\
& 32 & $4.61(-13)$ & $6.62(-13)$ & $-1.34(-12)$ & $7.64(-13)$ \\
& 64 & $1.01(-11)$ & $4.35(-12)$ & $-2.51(-11)$ & $1.61(-11)$ \\
\hline
\end{tabular}

we can represent the Legendre-collocation penalty scheme for (6.4):

$$
K_{\tau} \mathbf{u}^{\tau}=\lambda^{\tau} M \mathbf{u}^{\tau},
$$

where $\mathbf{u}^{\tau}=\left[\left(u_{-}^{\tau}\right)_{0}, \ldots,\left(u_{-}^{\tau}\right)_{N},\left(u_{+}^{\tau}\right)_{0}, \ldots,\left(u_{+}^{\tau}\right)_{N}\right]^{T}$.

The numerical computations are carried out for the case $\sigma_{1}^{\tau}=\sigma_{2}^{\tau}=\left\{\frac{2}{\pi} N(N+1)\right\}^{2}$ [11], [12], which is chosen for the matrix $K_{\tau}$ to be symmetric positive definite [9], [20]. The results, shown in Table 4, demonstrate the exponential rate of convergence. Simply replacing the set of points and the weights by the Chebyshev-Gauss-Lobatto points and weights, one can construct the multidomain Chebyshev-collocation penalty method, whose results also are provided in Table 4.

The theoretical analysis of the convergence for the multidomain spectral penalty method is left for future study, as is the analysis for optimizing the parameter $\tau_{i}$ for this eigenvalue problem.

7. Discussion. In this section we discuss the asymptotic behavior of the largest approximate eigenvalues obtained by the multidomain spectral techniques for the eigenproblem with a discontinuous coefficient. Figures 1-2 demonstrate the relative errors of the eigenvalues with fixed $N=4,8,16,32,64$ for each different method. The figures show that for the approximations with degree $N$, the fraction $\frac{2}{\pi}$ of the approximate eigenvalues converges to the analytic eigenvalues exponentially. Bernardi and Maday [2] and Vandeven [22] give rigorous proofs for finding the fraction of the approximate eigenvalues that approximate the eigenvalues of the second-order spectral differentiation operator.

We present the relative errors for the first 29 eigenvalues for a fixed $N=16$ in Table 5. One can see that $\frac{1}{\pi}$ of the eigenvalues approximate the analytic eigenvalues 

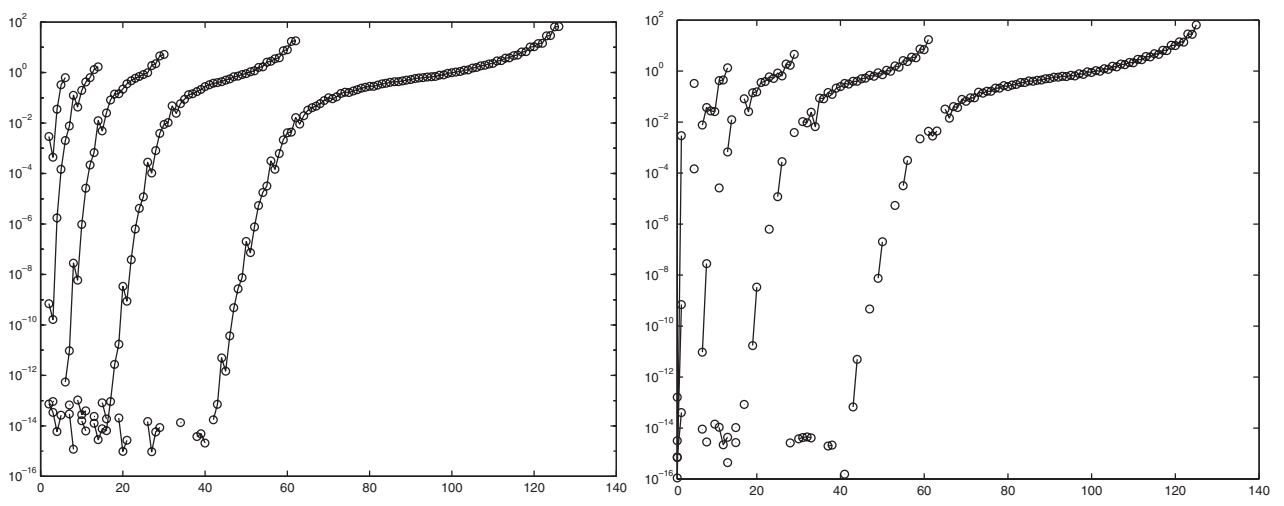

FIG. 1. Legendre-Galerkin method (left) and Legendre-collocation method 1 (right): the relative errors of all the eigenvalues for $N=4,8,16,32,64$.
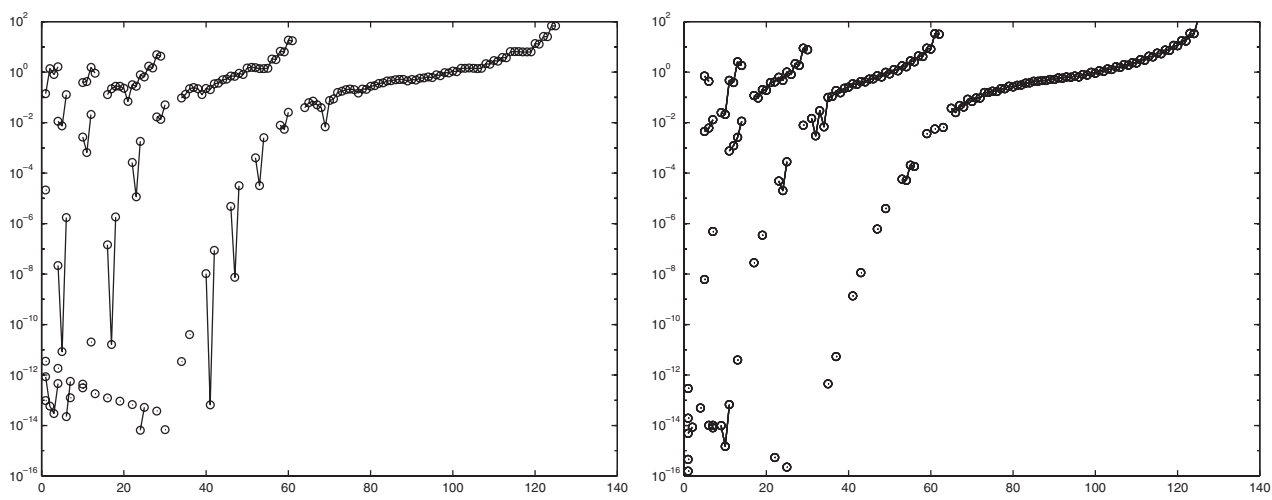

FIG. 2. Legendre-collocation method 2 (left) and Legendre-collocation penalty method (right): the relative errors of all the eigenvalues for $N=4,8,16,32,64$.

of the problem very accurately. One also can see that the Legendre-Galerkin method and the Legendre-collocation method 1 are more accurate than the other collocation methods. However, the Legendre-collocation method 2 and Legendre-collocation penalty method are relatively easier to implement because of their simplicity in dealing with the basis of the space used in the approximation.

8. Conclusion. In this paper, we have mainly discussed the Legendre-Galerkin, Legendre-collocation, and Legendre-collocation penalty methods with a domain decomposition approach in order to get exponentially accurate eigensolutions for a model eigenvalue problem with a piecewise continuous coefficient.

Acknowledgments. Special thanks to Wai-Sun Don, Jan Hesthaven, and Yvon Maday for their discussions and several suggestions regarding the topics in this paper. 
TABLE 5

Relative errors of eigenvalues for $\omega=2$ for the domain decomposition Legendre-Galerkin $(L G)$, Legendre-collocation method 1 (LC1), Legendre-collocation method 2 (LC2), Chebyshev-collocation method 2 (CC2), Legendre-collocation penalty method (LCP), Chebyshev-collocation penalty method $(C C P): \lambda_{l}^{*}$ is the lth approximate eigenvalue and $N=16$.

\begin{tabular}{|c||c|c|c|c|c|c|}
\hline \multicolumn{1}{|c|}{} & \multicolumn{7}{|c|}{$\left(\lambda_{l}^{*}-\lambda_{l}\right) / \lambda_{l}$} \\
\hline$l$ & LG & LC1 & LC2 & CC2 & LCP & CCP \\
\hline 1 & $-6.15(-15)$ & $-1.68(-14)$ & $-9.67(-14)$ & $8.55(-15)$ & $-1.05(-12)$ & $-1.79(-12)$ \\
2 & $-2.89(-14)$ & $-8.28(-16)$ & $-1.49(-13)$ & $-5.77(-14)$ & $-2.58(-14)$ & $4.47(-13)$ \\
3 & $-2.76(-16)$ & $4.69(-15)$ & $-8.33(-10)$ & $-2.28(-10)$ & $-8.31(-10)$ & $-2.26(-10)$ \\
4 & $-6.07(-15)$ & $1.14(-16)$ & $2.20(-08)$ & $6.13(-09)$ & $2.20(-08)$ & $6.15(-09)$ \\
5 & $5.52(-13)$ & $-1.42(-11)$ & $8.41(-12)$ & $-1.69(-08)$ & $-1.31(-08)$ & $-2.37(-08)$ \\
6 & $9.48(-12)$ & $9.48(-12)$ & $1.74(-06)$ & $4.80(-07)$ & $1.74(-06)$ & $4.81(-07)$ \\
7 & $2.83(-08)$ & $2.83(-08)$ & $-1.19(-04)$ & $-3.16(-05)$ & $-1.19(-04)$ & $-3.16(-05)$ \\
8 & $5.94(-09)$ & $-7.70(-08)$ & $-7.80(-05)$ & $-2.20(-05)$ & $-7.87(-05)$ & $-2.24(-05)$ \\
9 & $9.59(-07)$ & $-8.19(-06)$ & $-8.51(-04)$ & $-2.36(-04)$ & $-8.44(-04)$ & $-2.33(-04)$ \\
10 & $2.62(-05)$ & $2.62(-05)$ & $2.70(-03)$ & $7.56(-04)$ & $2.71(-03)$ & $7.61(-04)$ \\
11 & $2.15(-04)$ & $-1.11(-03)$ & $6.59(-04)$ & $1.20(-03)$ & $5.59(-04)$ & $1.16(-03)$ \\
12 & $6.74(-04)$ & $6.74(-04)$ & $2.11(-02)$ & $2.61(-03)$ & $2.11(-02)$ & $2.62(-03)$ \\
13 & $1.25(-02)$ & $1.25(-02)$ & $-2.07(-02)$ & $1.12(-02)$ & $-2.06(-02)$ & $1.12(-02)$ \\
14 & $4.88(-03)$ & $-1.50(-02)$ & $-6.98(-02)$ & $-3.63(-02)$ & $-7.02(-02)$ & $-3.65(-02)$ \\
15 & $2.53(-02)$ & $-2.96(-02)$ & $-6.49(-02)$ & $-4.14(-02)$ & $-6.42(-02)$ & $-4.10(-02)$ \\
16 & $8.25(-02)$ & $8.25(-02)$ & $1.29(-01)$ & $1.17(-01)$ & $1.30(-01)$ & $1.18(-01)$ \\
17 & $1.40(-01)$ & $2.55(-02)$ & $2.22(-01)$ & $9.57(-02)$ & $2.25(-01)$ & $9.61(-02)$ \\
18 & $1.44(-01)$ & $1.40(-01)$ & $2.76(-01)$ & $2.05(-01)$ & $2.76(-01)$ & $2.04(-01)$ \\
19 & $2.23(-01)$ & $1.53(-01)$ & $2.76(-01)$ & $1.92(-01)$ & $2.74(-01)$ & $1.91(-01)$ \\
20 & $3.57(-01)$ & $3.57(-01)$ & $2.30(-01)$ & $3.88(-01)$ & $2.27(-01)$ & $3.87(-01)$ \\
21 & $4.77(-01)$ & $3.89(-01)$ & $6.88(-02)$ & $4.15(-01)$ & $6.65(-02)$ & $4.15(-01)$ \\
22 & $5.89(-01)$ & $5.89(-01)$ & $3.17(-01)$ & $6.21(-01)$ & $3.18(-01)$ & $6.20(-01)$ \\
23 & $6.97(-01)$ & $5.20(-01)$ & $2.76(-01)$ & $4.69(-01)$ & $2.77(-01)$ & $4.69(-01)$ \\
24 & $8.38(-01)$ & $8.38(-01)$ & $7.77(-01)$ & $1.00(+00)$ & $7.78(-01)$ & $1.00(+00)$ \\
25 & $9.78(-01)$ & $6.44(-01)$ & $6.49(-01)$ & $8.09(-01)$ & $6.49(-01)$ & $8.09(-01)$ \\
26 & $1.89(+00)$ & $1.89(+00)$ & $1.74(+00)$ & $2.19(+00)$ & $1.75(+00)$ & $2.21(+00)$ \\
27 & $2.22(+00)$ & $1.67(+00)$ & $1.46(+00)$ & $1.86(+00)$ & $1.48(+00)$ & $1.88(+00)$ \\
28 & $4.59(+00)$ & $4.58(+00)$ & $4.96(+00)$ & $8.90(+00)$ & $4.99(+00)$ & $9.00(+00)$ \\
29 & $5.22(+00)$ & $3.93(+00)$ & $4.31(+00)$ & $7.82(+00)$ & $4.33(+00)$ & $7.91(+00)$ \\
\hline
\end{tabular}

\section{REFERENCES}

[1] W. Axmann and P. Kuchment, An efficient finite element method for computing spectra of photonic and acoustic band-gap materials: I. Scalar case, J. Comput. Phys., 150 (1999), pp. 468-481.

[2] C. Bernardi and Y. Maday, Spectral Methods, Handb. Numer. Anal. 5, North-Holland, Amsterdam, 1997.

[3] J. Broeng, D. Mogilevtsev, S. E. Barkou, and A. Buarklev, Photonic crystal fibres: A new class of optical waveguides, Opt. Fiber Technol., 1999.

[4] C. Canuto And A. Quarteroni, Error estimates for spectral and pseudospectral approximations of hyperbolic equations, SIAM J. Numer. Anal., 19 (1982), pp. 629-642.

[5] C. Canuto, M. Y. Hussaini, A. Quarteroni, and T. A. Zang, Spectral Methods in Fluid Dynamics, Springer Ser. Comput. Phys., Springer-Verlag, New York, 1988.

[6] D. C. Dobson, An efficient method for band structure calculations in $2 D$ photonic crystals, J. Comput. Phys., 149 (1999), pp. 363-376.

[7] D. Funaro and D. Gottlieb, A new method of imposing boundary conditions in pseudospectral approximations of hyperbolic equations, Math. Comp., 57 (1988), pp. 599-613.

[8] D. Funaro, Polynomial Approximation of Differential Equations, Springer-Verlag, New York, 1991. 
[9] G. H. Golub and C. Van Loan, Matrix Computations, Johns Hopkins University Press, Baltimore, MD, 1996.

[10] D. Gottlieb, M. Y. Hussaini, and S. A. Orszag, Theory and applications of spectral methods, in Spectral Methods for Partial Differential Equations, R. G. Voigt, D. Gottlieb, and M. Y. Hussaini, eds., SIAM, Philadelphia, 1984, pp. 1-54.

[11] D. Gottlieb And L. Lustman, The spectrum of the Chebyshev collocation operator for the heat equation, SIAM J. Numer. Anal., 20 (1983), pp. 909-921.

[12] J. S. Hesthaven, Integration preconditioning of pseudospectral operators. I. Basic linear operators, SIAM J. Numer. Anal., 35 (1998), pp. 1571-1593.

[13] J. S. Hesthaven and D. Gottlieb, Spectral Approximation of Partial Differential Equations: Numerical Analysis and Applications, Lecture Notes, Brown University, Providence, RI, 1996.

[14] J. D. Joannopoulos, R. D. Meade, And J. N. Winn, Photonic Crystals: Molding the Flow of Light, Princeton University Press, Princeton, NJ, 1995.

[15] S. John, Strong localization of photons in certain disordered dielectric superlattices, Phys. Rev. Lett., 58 (1987), pp. 2486-2489.

[16] S. G. Johnson And J. D. JoAnnopoulos, Block-iterative frequency-domain methods for Maxwell's equations in a planewave basis, Optics Express, 8 (2001), pp. 173-190.

[17] C. Mias, J. P. Webb, And R. L. Ferrari, Finite element modelling of electromagnetic waves in doubly and triply periodic structures, IEEE Proc.-Optoelectron, 145 (1999), pp. 111-118.

[18] M. S. Min And D. GotTLieb, On the convergence of the Fourier approximation for eigenvalues and eigenfunctions of discontinuous problems, SIAM J. Numer. Anal., 40 (2003), pp. 22542269.

[19] M. S. Min, Q. Y. Chen, And Y. Maday, Spectral method for $2 D$ photonic band structures, in Proceedings of the SPIE International Symposium on Integrated Optoelectronic Devices, SPIE, Washington, DC, 2004.

[20] Y. SAAD, Iterative Methods for Sparse Linear Systems, 2nd ed., SIAM, Philadelphia, 2003.

[21] G. Strang And G. Fix, An Analysis of the Finite Element Method, Prentice-Hall, Englewood Cliffs, NJ, 1973.

[22] H. VAndeVen, On the eigenvalues of second-order spectral differentiation operators, in Spectral and High Order Methods for Partial Differential Equations, in Proceedings of the ICOSAHOM '89 Conference, 1989.

[23] E. Yablonovitch, Inhibited spontaneous emission in solid-state physics and electronics, Phys. Rev. Lett., 58 (1987), pp. 2059-2062. 